\title{
TRANSPLANTE HEPÁTICO PARA SÍNDROMES HEPATOPULMONAR E DE OBSTRUÇÃO SINUSOIDAL
}

\section{Liver transplantation for hepatopulmonary and sinusoidal obstruction syndrome}

\author{
Maurício SILVA, Eduardo GARCIA, Tiago de CÁSTRIA, Roberta BARBOSA, \\ Fábio Luiz WAECHTER, Paulo Roberto Ott FONTES
}

ABCDDV/642

Silva M, Garcia E, Cástria T, Barbosa R, Waechter FL, Fontes PRO. Transplante hepático para síndromes hepatopulmonar e de obstrução sinusoidal. ABCD Arq Bras Cir Dig 2009;22(1):62-4

RESUMO - Introdução - A síndrome hepatopulmonar é complicação das doenças hepáticas que afeta a vascularização pulmonar, causando comprometimento do sistema respiratório e acompanha-se de elevada morbidade. O transplante hepático é o tratamento ideal para esses casos. O objetivo desse relato é descrever o caso de um paciente que apresentava síndrome hepatopulmonar avançada, devida à síndrome da obstrução sinusoidal. Relato do caso - Homem de 23 anos foi encaminhado com o diagnóstico de síndrome hepatopulmonar avançada e cirrose hepática sem etiologia idefinida. Exames de imagem foram compatíveis com shunt intrapulmonar, porém sem alterações da vascularização pulmonar factíveis de serem embolizadas. A endoscopia digestiva alta demonstrou varizes esofágicas de pequeno calibre. Exames laboratoriais para função hepática estavam alterados. O paciente foi submetido a transplante hepático falecendo 14 dias após o procedimento devido a complicações relacionadas à sepse $\mathrm{e}$ insuficiência ventilatória refratária. Conclusão - Na vigência de síndrome hepatopulmonar o único tratamento efetivo é o transplante hepático, para os casos em que não houver área de shunt factível de ser realizada a embolização seletiva.

DESCRITORES - Transplante hepático. Síndrome hepatopulmonar. Síndrome da obstrução sinusoidal.

\section{INTRODUÇÃO}

A síndrome hepatopulmonar (SHP) é caracterizada onde exista doença hepática predispondo à diminuição da função hepatocelular, hipertensão portal, dilatação vascular intrapulmonar e hipoxemia. Esta última caracterizada por $\mathrm{PaO} 2$ menor que $70 \mathrm{mmHg}$ ou $\mathrm{P}(\mathrm{A}-\mathrm{a}) \mathrm{O} 2$ maior que 20 $\mathrm{mmHg}$. Tendo em vista estes critérios, estima-se prevalência de $15 \%$ a $30 \%$ em candidatos a transplante ortotópico de fígado1. A patogenia dessa doença parece estar relacionada a desequilíbrio entre substâncias vasoconstritoras e vasodilatadoras dentro dos capilares pulmonares, e é considerada fator independente de risco para o aumento da mortalidade em pacientes cirróticos.

Em importante estudo prospectivo com 90 pacientes, verificou-se mortalidade de $33 \%$ em pacientes submetidos ao transplante ortotópico de fígado e portadores de SHP, enquanto o grupo sem comprometimento pulmonar apresentou mortalidade de 9,2\%2. $\mathrm{PaO} 2$ menor que 50 $\mathrm{mmHg}$ em ar ambiente, assim como quantificação de shunt intrapulmonar maior que $20 \%$ no estudo cintilográfico estão relacionados com mortalidade expressiva3. Tendo

Trabalho realizado no Servico de Pneumologia da Santa Casa de Porto Alegre, Programa de Pos-Graduacao em Hepatologia da Universidade Federal de Ciencias da Saude de Porto Alegre, Porto Alegre, RS, Brasil.

Endereço para correspondência: Mauricio Silva. Email: mauriciosilva11@yahoo.com.br em vista o prognóstico desfavorável dos pacientes com SHP, e a possível reversibilidade do quadro através do transplante ortotópico de fígado, esse segue sendo o tratamento ideal (fundamentalmente nos casos em que o estudo da vascularização pulmonar não demonstra áreas factíveis de serem tratadas através da radiologia intervencionista)1. Entretanto, não está claramente definido o momento em que ele deva ser contra-indicado.

Os autores relatam o caso de um paciente portador de SHP, submetido a esse procedimento, em que o comprometimento hepático foi devido à síndrome da obstrução sinusoidal.

\section{RELATO DO CASO}

Homem de 23 anos encaminhado por apresentar, há três anos, o diagnóstico de SHP avançada e cirrose hepática sem causa identificada. Não havia história de ascite ou hemorragia digestiva. Exame clínico revelou cianose, platipnéia, hipocratismo digital, icterícia, atrofia muscular e acentuado comprometimento ponderal (índice de massa corporal de $\left.16,2 \mathrm{Kg} / \mathrm{m}^{2}\right)$. A ecografia abdominal mostrou fígado com textura heterogênea, superfície irregular, veia porta medindo $1,3 \mathrm{~cm}$ e esplenomegalia. A angiotomografia computadorizada de tórax demonstrou extensas e difusas dilatações pré-capilares e capilares em ambos os campos pulmonares (Figura 1). Os achados da ecocardiografia com contraste e da arteriografia pulmonar 
foram compatíveis com shunt intrapulmonar, porém sem alterações da vascularização pulmonar factíveis de serem embolizadas. A endoscopia digestiva alta demonstrou varizes esofágicas de pequeno calibre. Os resultados das análises clínicas foram: albumina, $2,3 \mathrm{~g} / \mathrm{dL}$; atividade de protrombina, $42 \%$; bilirrubina total, $2 \mathrm{mg} / \mathrm{dL}$; hemoglobina, 17,4 g/dL; leucócitos 2410/ $\mu \mathrm{L}$ (72\% de neutrófilos); plaquetas, 17.000/ $\mu \mathrm{L} ; \mathrm{PaO} 2,29 \mathrm{mmHg}$; saturação de O2/ $\mathrm{Hb}, 53 \%$.

Após administração de $\mathrm{O} 2$ a $100 \%$ a $\mathrm{PaO} 2$ mudou para $43,7 \mathrm{mmHg}$ e saturação de $\mathrm{O} 2 / \mathrm{Hb}$ para $77 \%$.

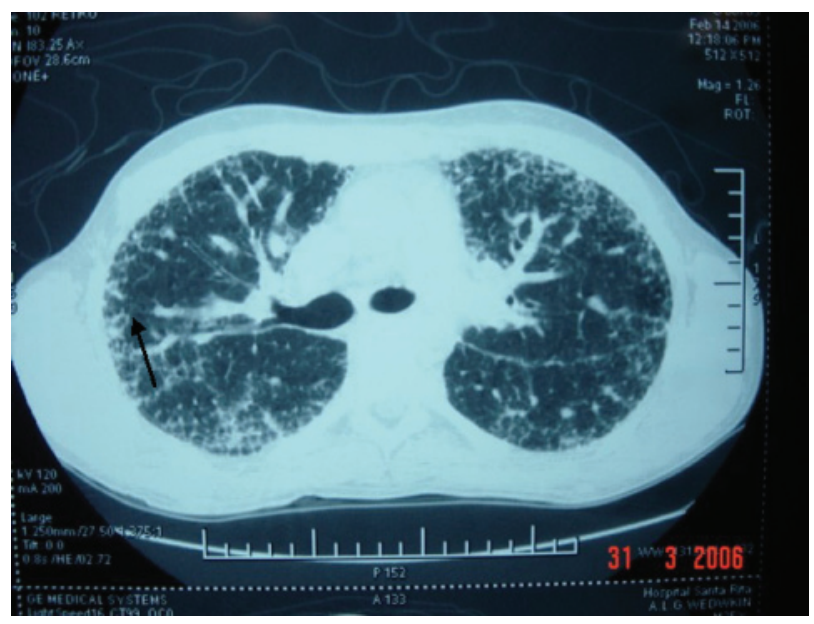

FIGURA 1 - Extensas e difusas dilatações capilares e précapilares nos campos pulmonares bilateralmente

Deste modo, realizou-se o diagnóstico presuntivo de cirrose hepática em fase avançada e SHP grave, e extensa discussão sobre os riscos relacionados ao transplante, que foi realizado vindo o paciente a falecer 14 dias após o procedimento devido a complicações relacionadas à sepse e insuficiência ventilatória refratária. O estudo histopatológico revelou a presença de síndrome da obstrução sinusoidal (Figura 2).

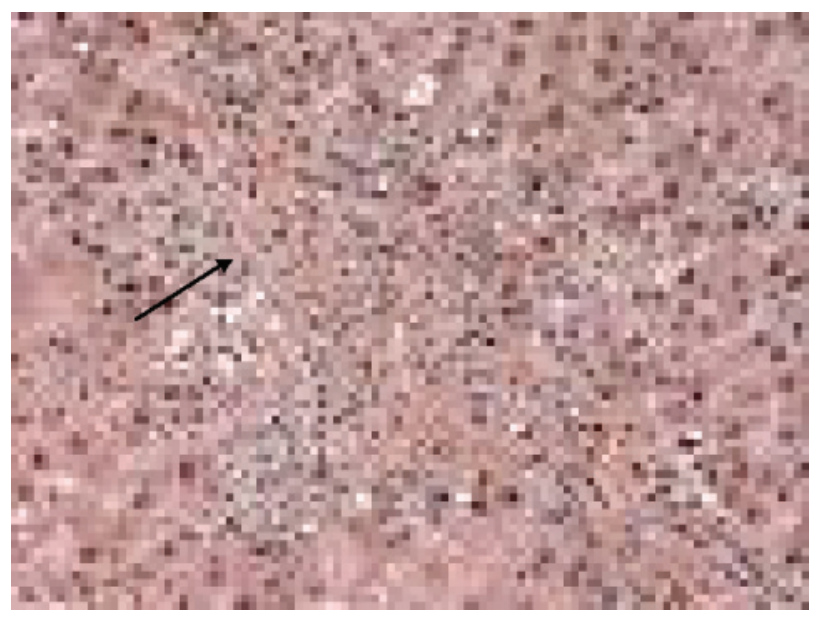

FIGURA 2 - Dilatação sinusoidal sem a presença de transformação nodular

\section{DISCUSSÃO}

Kennedy e Knudson, em 1977, foram os primeiros a descreverem a SHP, caracterizando-a como a associação entre disfunção hepática e respiratória, devido a dilatações na vascularização pulmonar4. O quadro clínico típico é caracterizado por dispnéia, hipoxemia crônica e progressiva, cianose e hipocratismo digital. Além disso, a presença de platipnéia e de ortodeoxia sugere o diagnóstico.

Estudos demonstram que o transplante é a única terapia eficaz para ela, tendo impacto significativo na sobrevida, podendo levar a resolução completa da doença2,3. Em 2003, Arguedas et al demonstraram sobrevida diminuída em pacientes com SHP após serem submetidos ao transplante3. Identificou-se que a medida de $\mathrm{PaO} 2$ e a quantificação do shunt intrapulmonar são testes pré-operatórios que podem ser feitos a fim de avaliar o risco do procedimento nesses pacientes. $\mathrm{PaO} 2$ menor que $50 \mathrm{mmHg}$ e estudo cintilográfico demonstrando shunt intrapulmonar maior que $20 \%$ foram 7,5 vezes mais frequentes nos pacientes com SHP que foram a óbito após o transplante3.

Com o intuito de auxiliar a avaliação de pacientes que poderão ser beneficiados, Bottari et al propuseram um modelo que mensura, no período pré-operatório, o risco relacionado ao transplante, considerando o escore Child-Pugh-Turcotte e o grau de hipoxemia5. Esse modelo permite que a intervenção no curso da doença ocorra em estágio mais precoce, diminuindo o risco de mortalidade pós-operatória.

Sabe-se que a SHP está relacionada com qualquer hepatopatia que curse com hipertensão portal, embora mais frequentemente esteja associada à cirrose1. No presente caso, a causa da hipertensão portal foi a síndrome da obstrução sinusoidal. Essa doença se caracteriza pela obstrução da micro-circulação hepática, tendo como apresentação clássica a hepatomegalia dolorosa, hiperbilirrubinemia e ascite. Apresenta causa variada, podendo estar relacionada a agentes quimioterápicos, imunossupressores, chás de ervas exóticas, entre outros6.

Frequentemente o diagnóstico presuntivo de cirrose é baseado em um contexto de achados que dispensam o exame histopatológico; porém, em algumas situações, seu diagnóstico pode não estar correto. Esse é o caso do presente relato, em que o diagnóstico, realizado em um primeiro momento foi, posteriormente, descartado. Ressalta-se que o transplante é o tratamento de escolha da hipertensão portal grave e da insuficiência hepática avançada, independente da causa da doença, naquelas situações onde possa haver reversibilidade após o procedimento.

\section{CONCLUSÃO}

$\mathrm{Na}$ vigência de síndrome hepatopulmonar o único tratamento efetivo é o transplante hepático, para os casos em que não houver área de shunt factível de ser realizada a embolização seletiva 
Silva M, Garcia E, Cástria T, Barbosa R, Waechter FL, Fontes PRO. Liver transplantation for hepatopulmonary and sinusoidal obstruction syndrome. ABCD Arq Bras Cir Dig 2009;22(1):62-4

ABSTRACT - Introduction - The hepatopulmonary syndrome is a complication of liver diseases that affects the pulmonary vascular system compromising respiratory function. High morbidity rates are associated with this syndrome. Liver transplantation is the treatment of choice. The aim of this report is to present the case of a patient who sustained advanced hepatopulmonary syndrome resulting from the sinusoidal obstruction syndrome. Case report-A 23-year-old male was referred with the diagnosis of advanced hepatopulmonary syndrome and liver cirrhosis of undefined etiology. Imaging studies were consistent with intrapulmonary shunt yet without alterations in pulmonary vascularization amenable to embolization. Upper digestive endoscopy demonstrated small-caliber esophagian varices. Laboratory tests for liver function showed alterations. The patient underwent liver transplantation and died 14 days after, due to sepsis-related complications and refractory ventilatory failure. Conclusion - In cases when it is not feasible to perform selective embolization in the shunt area, the only effective treatment for hepatopulmonary syndrome is liver transplantation.

HEADINGS - Liver transplantation. Hepatopulmonary syndrome. Sinusoidal obstruction syndrome.

\section{REFERÊNCIAS}

1. Palma DT, Fallon MB. The hepatopulmonary syndrome, J Hepatol 2006;45:61725 .

2. Schenk P, Fuhrmann V, Madl C, Kunf G, Lehr S, Kandel O, Müller C. Hepatopulmonary syndrome: prevalence and predictive value of various cut-offs for arterial oxygenation and their clinical consequences. Gut 2002;51:853-9.

3. Arguedas GA, Abrams MJ, Krowka J, Fallon MB. Prospective evaluation of outcomes and predictors of mortality in patients with hepatopulmonary syndrome undergoing liver transplantation. Hepatology 2003;37:192-7.

4. Kennedy TC, Knudson RJ. Exercise-aggravated hypoxemia and orthodeoxia in cirrhosis. Chest 1977;72:305-9.
5. Bottari G, Mazzeo AT, Santamaria LB. The importance of predicting the prognosis in patients with hepatopulmonary syndrome: a simple scoring system. Transplant Proc 2006;38:795-7.

6. LeLeve LD, Shulman HM, Mc Donald GB. Toxic injury to hepatic sinuoids: sinusoidal obstruction syndrome (veno-occlusive disease). Semin Liver Dis $2002 ; 22: 27-42$

Fonte de financiamento: não há Conflito de interesse: não há Recebido para publicação: 30/07/2008 Aceito para publicação: 27/11/2008 